Relations industrielles

Industrial Relations

\title{
Berggren, Christian, Alternatives to Lean Production. Work Organization in the Swedish Auto Industry
}

\section{Martin Dumas}

Volume 48, numéro 2, 1993

URI : https://id.erudit.org/iderudit/050865ar

DOI : https://doi.org/10.7202/050865ar

Aller au sommaire du numéro

Éditeur(s)

Département des relations industrielles de l'Université Laval

ISSN

0034-379X (imprimé)

1703-8138 (numérique)

Découvrir la revue

Citer ce compte rendu

Dumas, M. (1993). Compte rendu de [Berggren, Christian, Alternatives to Lean Production. Work Organization in the Swedish Auto Industry]. Relations industrielles / Industrial Relations, 48(2), 374-376.

https://doi.org/10.7202/050865ar

Tous droits réservés @ C Département des relations industrielles de l'Université Laval, 1993
Ce document est protégé par la loi sur le droit d'auteur. L'utilisation des services d'Érudit (y compris la reproduction) est assujettie à sa politique d'utilisation que vous pouvez consulter en ligne.

https://apropos.erudit.org/fr/usagers/politique-dutilisation/ 
Nécessaire initiation à une loi internationale, indissociablement liée aux droits fondamentaux de la personne, « dont l'autorité transcende les frontières » (p. 85).

Pierre VerGe

Université Laval

Alternatives to Lean Production. Work Organization in the Swedish Auto Industry, by Christian Berggren, Ithaca, New York, ILR Press, 1992, 286 p., ISBN 0-87546-193-X

Lorsque Womack, Jones et Roos publiaient, en 1990, The Machine that Changed the World, un avertissement était lancé : l'industrie occidentale court à sa perte si elle ne fait pas siennes les techniques de production contractile (lean production). Pensées, développées et appliquées au Japon, ces techniques étaient décrites comme les seules unissant à la fois les avantages de la production de masse (e.g. réduction des coûts de production) et ceux de la production artisanale (e.g. qualité du produit). L'on pronostiquait alors une véritable révolution qui ferait du modèle de production japonais un patron standard destiné à marquer le XXI ${ }^{c}$ siècle. De ce contexte, la contribution de Berggren tire l'essentiel de sa pertinence. L'auteur conteste en effet les avancées de Womack, Jones et Roos et cherche à contrecarrer un courant qu'il qualifiera de propagandiste.

Puisant dans de riches matériaux empiriques (six monographies d'entreprises de l'industrie automobile suédoise réalisées par l'auteur font l'objet d'une attention particulière et sont complétées par quatorze autres regroupant le Japon, les États-Unis et quelques pays d'Europe), Berggren offre une vision différente et critique du modèle japonais d'une part et, d'autre part, présente une variété de modèles d'organisation technique et sociale du travail qui contrastent avec le premier sans se mouler à la production de masse ou artisanale. En outre, il cherche à évaluer les différentes possibilités de concilier les exigences de la production avec les capacités et besoins de l'homme.

Contrairement aux dires de nombreux analystes dont Krafcik et MacDuffie, Berggren soutient qu'il n'existe pas de rupture entre le taylorisme et le toyotisme; le second constituerait plutôt selon lui une extension complexe du premier. Pour appuyer sa thèse, il explique dans les moindres détails les différences et similitudes caractérisant les deux modes de production comparés et en retient trois éléments importants. Le premier renvoie au caractère hautement standardisé des deux systèmes de production ainsi qu'au maintien solide des prérogatives patronales. Le second a trait à l'importance du rôle du contremaître et à la faible portée des recommandations syndicales dont le contremaître se fait d'ailleurs le héraut. Le troisième élément, traité avec plus de nuances, concerne les relations entre le salarié et la compagnie. Berggren y voit une continuation et un changement en regard du taylorisme dans la mesure où le toyotisme parvient à favoriser l'identification des travailleurs vis-à-vis de la compagnie par le biais de politiques individuelles.

Quant aux solutions de rechange au modèle de production contractile japonais, l'auteur nous propose une variété de modèles, étudiés en Suède, qui se situent aux 
croisées de deux continuums, soit l'organisation du travail humain (individu vs équipe de travail intégrée et qualifiée) et l'organisation technique (chaîne de production traditionnelle vs assemblage intégré). À l'intérieur de cette grille, de nombreuses combinaisons sont possibles et ont d'ailleurs fait l'objet d'essais, plus particulièrement chez Volvo. C'est pourquoi l'auteur rejette toute référence au soi-disant « modèle Volvo ", rappelant, et c'est là sa principale conclusion, qu'il est trompeur de prétendre à l'universalité en matière d'organisation du travail et qu'à cet égard le suivi d'un modèle unique de production est impensable. Il soutiendra en contrepartie qu'il est plus pertinent et exact de considérer les différents modes d'organisation du travail comme des synthèses d'autres modes tout aussi caractéristiques et comme les résultantes de facteurs exogènes particuliers. Sa thèse s'appuie en partie sur les pressions diverses exercées par des forces externes sur les entreprises de toutes sortes. Les taux de chômage peu élevés et la forte influence des syndicats ont été à l'origine des multiples formes de réorganisation du travail en Suède. L'historique de l'usine Uddevalla, exemple achevé d'une organisation de type intégré, nous montre qu'un nouveau mode de production redonnant au travailleur sa dignité fut introduit dans l'espoir de recruter plus facilement de bons employés.

De manière à circonscrire mieux l'une des solutions de remplacement du toyotisme, l'auteur brosse un tableau synthèse où la « stratégie anthropocentrique " suédoise est comparée à celle développée au Japon. D'une manière générale, le modèle suédois se distingue du modèle nippon en regard de l'autonomie réelle qu'il accorde aux salariés, de la démocratie appliquée au choix du chef d'équipe (contremaître), de la contractualisation des objectifs de performance (par voie de négociation) et de l'omniprésence syndicale.

Le lecteur trouvera en outre dans Alternatives to Lean Production de nombreux arguments, solidement enracinés, en faveur de l'humanisation des pratiques de travail, du contrôle ouvrier et du respect des différences individuelles. A cet égard, argumente l'auteur, certaines usines de l'industrie automobile suédoise emploient près de $40 \%$ de femmes. Considérant la nature des tâches effectuées, cette assertion est néanmoins trompeuse en ce qu'elle suggère l'existence d'un lien unissant logiquement l'embauche de femmes et l'amélioration des conditions de travail. L'analyse ergonomique rejette en effet les distinctions catégorielles ainsi que les raisonnements a priori de ce type. Par ailleurs, le volume renferme de nombreux tableaux et schémas dont certains, et plus particulièrement ceux illustrant différents plans d'organisation technique, s'avèrent indispensables à une juste représentation de nombreuses désignations techniques.

Enfin, le volume se subdivise en treize chapitres qui se succèdent malheureusement d'une manière très peu orthodoxe. Par exemple, le chapitre méthodologique vient entrecouper la présentation des matériaux empiriques et la disposition d'autres chapitres, notamment celui faisant état des aspects abrutissants de la ligne d'assemblage, est contestable. Toutefois, les nombreux sous-titres et un index exhaustif facilitent le repérage d'éléments d'analyse de tous gentes.

Le lecteur intéressé par les différentes formes d'organisation du travail et les enjeux économiques et sociaux qui y sont associés aura l'occasion de suivre dans l'ouvrage de Berggren une pensée critique et originale fondée sur l'analyse de matériaux 
empiriques. Alternatives to Lean Production offre également une remarquable contribution au monde scientifique en regard de sa dimension comparative internationale.

Martin Dumas

Université Laval

Merit Pay: Linking Pay Increases to Performance Ratings, by Robert L. Heneman, Reading, MA., Addison-Wesley Publishing Co., 1992, 298 p., ISBN 0-201-52504-6

Les systèmes de rémunération liant les augmentations de salaires à la performance individuelle ou collective sont utilisés par les employeurs dans le but d'augmenter la motivation et la productivité des personnes à leur emploi. L'ouverture à des marchés plus vastes et plus compétitifs accentue l'importance de la productivité des entreprises.

Le livre de Robert L. Heneman traite principalement de la rémunération individuelle au mérite. Les objectifs de l'auteur sont doubles. Premièrement, présenter un cadre théorique de référence pour toute personne qui désire faire un rapide tour d'horizon des différents plans de rémunération au mérite qui sont appliqués le plus fréquemment dans les organisations. Deuxièmement, faire ressortir les apects pratiques qui doivent être considérés dans le processus de révision de la politique de rémunération d'une organisation pour accorder une plus grande importance à la performance individuelle. À mon sens, ce volume constitue un excellent guide pour un employeur qui voudrait s'engager dans cette direction.

Heneman définit la rémunération individuelle au mérite comme étant à la fois une formule de récompense et une mesure incitative. Il présente donc la rémunération au mérite comme une méthode qui sert à mesurer la performance antérieure d'une personne en vue d'influencer son comportement futur pour obtenir un niveau attendu de performance. Ce faisant, il procède à une énumération exhaustive des programmes de rémunération liée à la performance appliqués dans les entreprises à succès aux États-Unis et compare les programmes de rémunération basés sur le mérite individuel avec ceux basés sur le mérite collectif. Il examine aussi les effets de la rémunération au mérite individuel sur une longue période de temps, les distinguant ainsi des programmes collectifs dont les effets sont la plupart du temps limités dans le temps.

Le volume comprend sept chapitres et une bibliographie complète et à jour. Les deux premiers chapitres sont surtout consacrés à présenter une revue de la théorie derrière la rémunération liée à la performance et de la recherche actuelle dans ce domaine. Heneman y présente principalement deux théories qui sont à l'origine de ces programmes. La première est du domaine du comportement organisationnel; l'auteur fait le point sur les recherches qui ont examiné le lien entre la rémunération liée à la performance individuelle et l'augmentation de la motivation. La deuxième théorie plutôt du domaine économique considère l'augmentation de la performance comme un des facteurs qui influent sur l'augmentation de la productivité de l'organisation. 